\title{
GERAÇÃO DE SINAIS DE RF POR MISTURA DE HARMÔNICAS DE PORTADORAS ÓPTICAS MODULADAS EM AMPLIFICADORES ÓPTICOS SEMICONDUTORES
}

\author{
A. C. Bordonalli, J. L. Benitez, J. A. Guimarães, C. M. Gallep e E. Conforti
}

\begin{abstract}
Resumo - A propriedade do ganho cruzado de amplificadores ópticos semicondutores visando a geração de sinais de RF é investigada experimentalmente neste trabalho. Observou-se que a utilização de amplificadores śpticos altamente saturados e realimentados opticamente วode ter desempenho similar ao caso de vários amplificadores em cascata nas aplicações de geração de sinais de RF, oferecendo um ganho acima de $10 \mathrm{~dB}$, para duas portadoras ópticas moduladas e separadas de $8 \mathrm{~nm}$. A saturação altera o mecanismo de ganho do amplificador, produzindo um efeito de mistura entre as harmônicas de modulação dos sinais ópticos. Para portadoras ópticas moduladas em $250 \mathrm{MHz}$ e $400 \mathrm{MHz}$, produziu-se, após a iotodeteção, componentes eletrônicas com frequiências de batimento de 150 e $650 \mathrm{MHz}$.
\end{abstract}

Abstract - The gain-crossed property of semiconductor optical amplifiers is experimentally investigated. It was observed that optically feed-backed saturated amplifiers could achieve similar performance as that of cascaded amplifiers, offering up to $10 \mathrm{~dB}$ gain to two 8-nm-appart nodulated optical carriers. Due to saturation, the amplifier sain mechanism is altered. leading to a mixing effect that causes the frequency components of the optical signals to be exchanged. For optical carriers modulated at $250 \mathrm{MHz}$ and $100 \mathrm{MHz}$. this mixing effect produced, after photodetection, up and down-converted electronic components at 150 and $650 \mathrm{MHz}$, respectively.

Palavras-chave: Amplificadores ópticos semicondutores, Eeração de sinais, sistemas ópticos.

\section{INTRODUÇÃO}

Recentemente, a geração fotônica de sinais de RF, microondas e ondas milimétricas tem sido amplamente estudada. Estas técnicas usam a fibra óptica como meio de transmissão para portadoras de considerável estabilidade, com aplicações em sistemas de comunicações móveis [1-2], sistemas de comunicações via satélite [3] e sistemas de subportadoras multiplexadas [4]. Técnicas como enlace travamento de fase óptica [5-6], travamento por injeção [7] e travamento de modos [8] têm demonstrado a possibilidade de conversão optoeletrônica e a geração de portadoras de RF e microondas.

O enlace de travamento de fase óptica (optical phaselock loop - OPLL) é uma técnica baseada nos enlaces de travamento de fase eletrônicos (phase-lock loop - PLL),

Os autores são da UNICAMP - FEEC - Departamento de Microondas e Óptica - CEPOF - Centro de Pesquisas em Óptica e Fotônica. E-mail: aldario@dmo.fee.unicamp.br. promovendo a conversão de um sinal eletrônico (em uma dada freqüência) para o domínio óptico. O PLL é uma técnica bem estabelecida em eletrônica e permite a sincronização de uma fonte local à freqüência (e fase) de um sinal piloto. O funcionamento do OPLL é análogo ao do PLL. A Fig. 1 mostra o diagrama esquemático de um OPLL heteródino. Neste sistema, utilizam-se dois lasers, um chamado de mestre. que faz o papel de emissor e um outro, chamado de escravo. que faz analogia ao papel de oscilador local de enlaces de travamento de fase eletrônicos. O objetivo desse tipo de sistema é manter o valor da diferença de freqüência entre os lasers igual à frequiência do sinal eletrônico que se deseja converter para o domínio óptico (representado pela fonte de offset). A capacidade do circuito de executar esta tarefa (faixa de sintonia) depende das características do circuito de realimentação. Para que o travamento seja alcançado, os sinais dos lasers são sobrepostos na região ativa de um fotodetetor. Isto gera um sinal eletrônico de batimento numa freqüência intermediária (FI) igual à diferença de freqüência entre os dois lasers. $\mathrm{O}$ sinal de FI é então misturado com um sinal gerado por um gerador de offset em um detetor de fase (mixer). Este detetor de fase compara as fases do sinal de batimento e do sinal de offset. o último operando em uma frequiência próxima àquela do sinal de FI. Um sinal de erro de fase é gerado na saída do detetor de fase. Este sinal é processado pelo lïltro do enlace e alimenta o laser escravo, de forma a minimizar o erro de fase no ciclo seguinte. O enlace é considerado travado quando a freqüiencia de operação do laser escravo é controlada de forma a manter a freqüência de FI (diferença de freqüência entre os lasers) constante e igual à freqüência de offset. Uma vez convertido para o domínio óptico, o sinal eletrônico pode ser recuperado após a fotodeteção do sinal de saída do OPLL no ponto de destino.

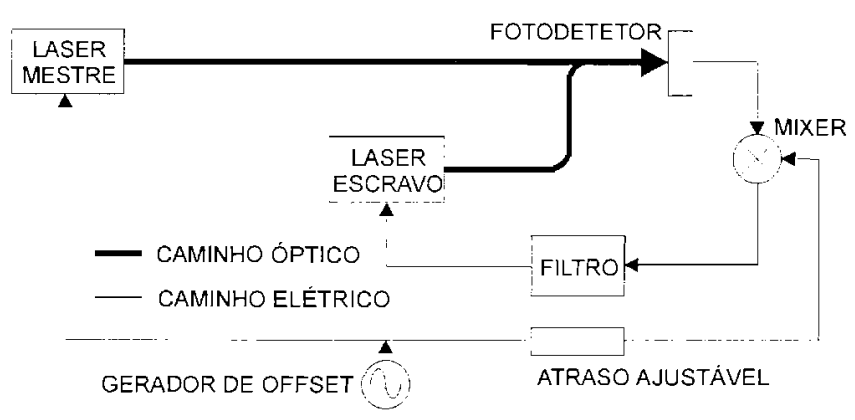

Figura 1. Diagrama esquemático de um OPLL.

A realização de OPLLs compactos e de baixo custo necessita de lasers semicondutores sem técnicas externas de estreitamento de linha. Devido ao fato de que o sinal de erro de fase é o sinal de controle do enlace, sinal este que contém o espectro do ruído de fase dos lasers, a largura de 
banda do enlace deve ser suficiente para cobrir a faixa de frequiência deste ruído. Assim, surgem os principais fatores de limitação no projeto prático do OPLL, que vão desde a limitação da largura de linha de fontes ópticas (abaixo de alguns $\mathrm{MHz}$ ) até o tempo de atraso do enlace de realimentação (que deve ser mantido abaixo de alguns ns). Para atender os pré-requisitos de projeto, o custo de fontes ópticas especiais e de circuitos optoeletrônicos com baixo tempo de atraso pode inviabilizar a aplicação destes sistemas. A Fig. 2 mostra o espectro eletrônico gerado após a fotodeteção dos sinais ópticos do OPLL [5], onde os lasers possuíam cerca de $2,5 \mathrm{MHz}$ de largura de linha e tempo de atraso do circuito de realimentação estimado em cerca de 3 ns. Notar que a frequêencia central do sinal é de $5,1 \mathrm{GHz}$. Neste caso, a medida da densidade espectral de ruído de fase produz um valor melhor que $-76 \mathrm{dBc} / \mathrm{Hz}$ em $20 \mathrm{MHz}$ de offset.

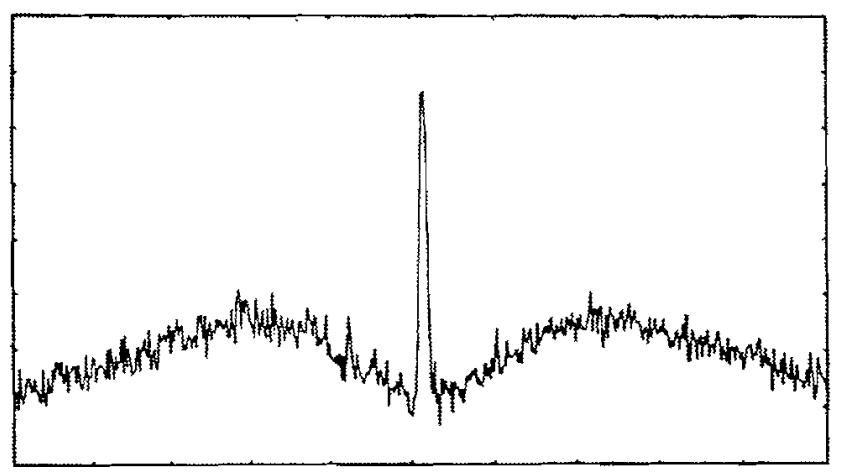

Figura 2. Espectro de sinal eletrônico obtido após fotodeteção do sinal de saída de um OPLL [5]. Largura de banda de resolução $(\mathrm{RBW})=300 \mathrm{kHz}$; referência $=-5 \mathrm{dBm}$; eixo vertical $=5 \mathrm{~dB} /$ div.; , eixo horizontal $10 \mathrm{MHz} /$ div.: freqüência central $=5 \mathrm{GHz}$.

Uma outra técnica de geração/conversão de sinais por métodos optoeletrônicos é o travamento por injeção óptica (optical injection locking - OIL) [6,7]. A Fig. 3 mostra o diagrama de blocos de um sistema OIL. Neste caso, o travamento da freqüência dos lasers ocorre devido à injeção de luz do laser mestre na regiāo ativa do laser escravo. As variaçōes nos mecanismos de ganho do laser escravo, induzidas pela injeção da luz do laser mestre, forçam o laser escravo a operar na mesma freqüência do laser mestre. Apesar de ser uma técnica homódina. podem-se alterar as condições de funcionamento do laser mestre, de forma a se induzir a operação heteródina. A Fig. 4 mostra o diagrama esquemático de um OIL que permite a geração/conversão de sinais eletrônicos no/para o domínio óptico. A luz do laser mestre é modulada em uma freqüência correspondente a uma sub-harmônica da frequiência do sinal eletrônico de interesse. Como conseqüência, o sinal modulado do laser mestre irá possuir, além da fundamental, harmônicas de modulação. Na seqüência, o sinal modulado do laser mestre é dividido e injetado em dois lasers escravos independentes. Após a injeção. os lasers escravos são forçados a travar em relação as harmônicas de modulação do laser mestre. Desta forma, dependendo das harmônicas de modulação do sinal do laser mestre escolhidas para o travamento, os dois lasers escravos operarão em frequiências ópticas distintas, porém, com a diferença entre elas igual ao valor da freqüência do sinal eletrônico de interesse. Se o sinal dos dois lasers são sobrepostos na região ativa de um fotodetetor, o sinal eletrônico resultante irá possuir a freqüência desejada.

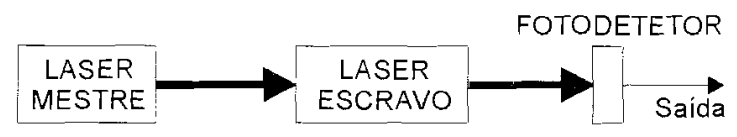

Figura 3. Diagrama esquemático da técnica OIL.

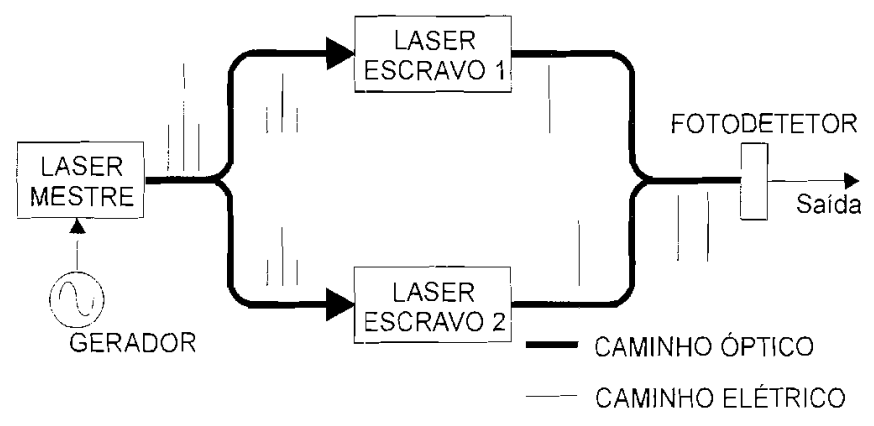

Figura 4. Diagrama esquemático de um OIL heteródino.

A Fig. 5 mostra o espectro eletrônico gerado após a fotodeteção do sinal de saída do sistema OIL descrito acima [7]. Notar que a freqüência central do sinal é de cerca de 35 $\mathrm{GHz}$. Neste caso, a medida da densidade espectral de ruído de fase produz um valor melhor que $-65 \mathrm{dBc} / \mathrm{Hz}$ em $400 \mathrm{~Hz}$ de offset.

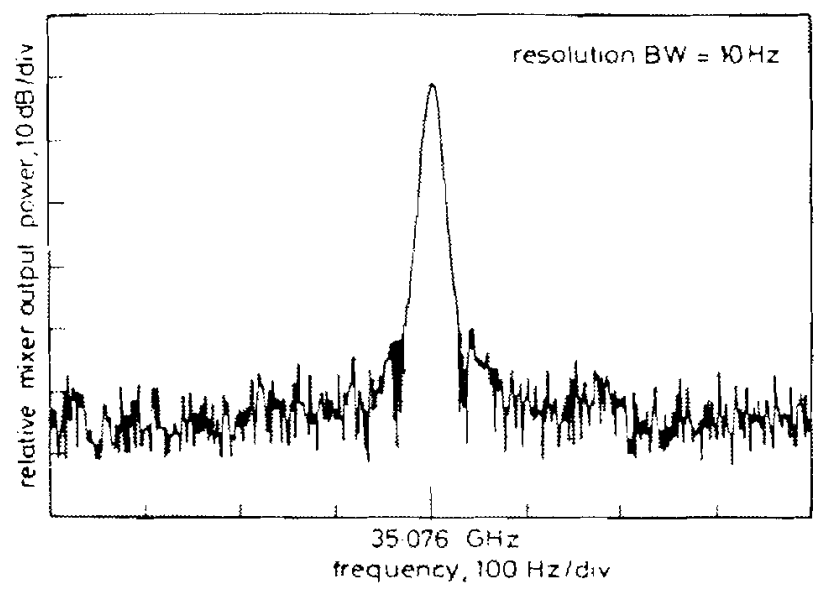

Figura 5. Espectro de sinal eletrônico obtido após fotodeteção do sinal de saída de um OIL [7].

Infelizmente, o OIL também apresenta desvantagens. A faixa de travamento do OIL é limitada (< a do OPLL), o que requer um controle muito preciso de aproximação das freqüências das fontes. O controle efetivo do erro de fase depende dos níveis de injeção, exigindo lasers mestre de alta potência de saída, ou, se estes não estiverem disponíveis, a pré-amplificação do sinal óptico. Como não existe compensação por realimentação eletrônica, o OIL não consegue manter uma referência de fase, sendo que variações em temperatura e corrente podem causar alterações na supressão de ruído de fase e instabilidades.

Uma técnica mais recente utiliza o OPLL e OIL de uma forma combinada, formando o enlace de travamento de fase 
com injeção óptica (optical injection phase-locked loop OIPLL) [6]. Neste sistema, Fig. 6, parte da luz emitida pelo laser mestre é modulada por uma fonte de offset e injetada no laser escravo. A outra parte é combinada com o sinal de saída do laser escravo. O fotodetetor fornece o sinal de batimento entre os dois lasers, produzindo em sua saída um sinal eletrônico de frequiência intermediária (FI) igual à diferença de freqüência entre os dois lasers. O sinal de FI é então misturado com um sinal gerado pelo mesmo gerador de offset que modulou uma amostra do sinal do laser mestre, em um detetor de fase (mixer). Este detetor de fase compara as fases do sinal de batimento e do sinal de offset, o último operando em uma freqüência próxina àquela do sinal de FI. Im sinal de erro de fase é gerado na saída do detetor de fase. Este sinal é processado pelo filtro do enlace e alimenta laser escravo de forma a minimizar o erro de fase no ciclo seguinte. $O^{-}$enlace é considerado travado quando a frequência de operação do laser escravo se iguala àquela da banda lateral de modulação do laser mestre e é controlada de forma a manter a freqüência de FI constante e igual à frequiência de offset. Uma vez convertido para o domínio óptico, o sinal eletrônico pode ser recuperado após a fotodeteção do sinal de saída do OIPLL no ponto de destino. A principal contribuição da injeção de luz na utilização combinada do OPLL com o OIL é a de controlar o ruído de erro de fase para altas frequiências. Dessa forma, a largura de banda dos enlaces de realimentação pode ser estreita, quando comparada com a largura de linha dos lasers, ao mesmo tempo que a utilização de lasers comerciais, com larguras de linha consideráveis, é possível, mesmo para sistemas com longo tempo de atraso. A contribuição da realimentação é a de compensar as variações de erro de fase de baixa freqüência, criando uma referência de fase que possibilita a sintonia do sistema em uma faixa de frequiência que depende somente das características do enlace de realimentação. Assim. para uma dada configuração de filtro. o OIPLL possui espectro de erro de fase que é uma combinação dos espectros de erro de fase dos sistemas OIL e OPLL equivalentes.

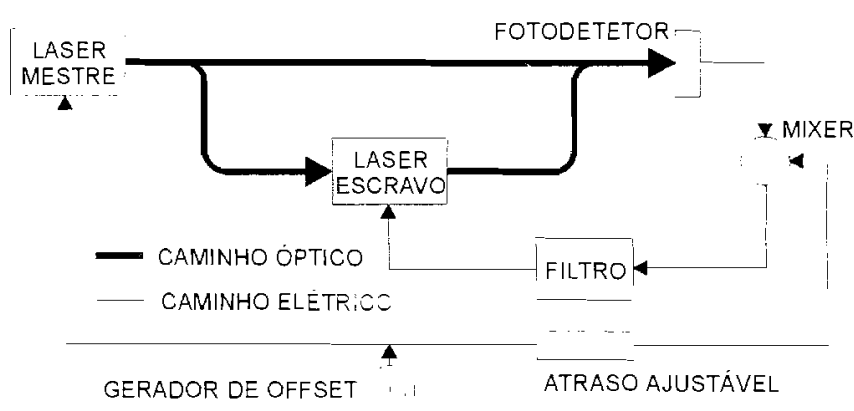

Figura 6. Diagrama esquemático de um OIPLL.

A Fig. 7 mostra o espectro eletrônico gerado após a fotodeteção dos sinais ópticos do OIPLL [6], onde os lasers possuíam cerca de $10 \mathrm{MHz}$ de largura de linha e tempo de atraso do circuito de realimentação estimado em cerca de 20 ns. Notar que a freqüência central do sinal é de $8 \mathrm{GHz}$. Neste caso, a medida da densidade espectral de ruído de fase produz um valor melhor que $-94 \mathrm{dBc} / \mathrm{Hz}$ em $10 \mathrm{kHz}$ de offset. Como as outras técnicas, o OIPLL também apresenta desvantagens. Para atender os pré-requisitos de projeto. deve-se controlar com precisão os caminhos elétricos e ópticos do OIPLL, de modo a evitar diferenças nas comparações de fase feitas pela parte OIL e OPLL do sistema. Uma maneira de se alcançarem os níveis de precisão exigidos (da ordem de comprimentos de onda da luz) é através da utilização de estruturas optoeletrônicas integradas (optoelectronic integrated circuit - OEIC), que são, geralmente, de alto custo.

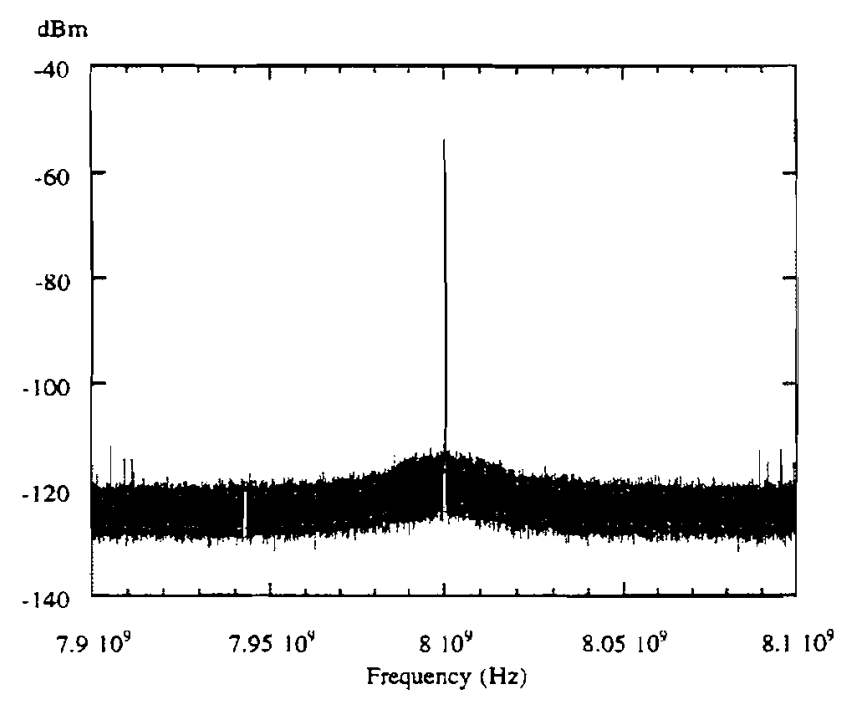

Figura 7. Espectro de sinal eletrônico obtido após fotodeteção do sinal de saída de um OIPLL [6].

A última técnica de geração/conversão de sinais mencionada anteriormente foi o travamento de modos (mode locking - ML). Está técnica consiste em sincronizar o padrão de fase dos modo internos de um laser, de forma que o mesmo passa a operar em um regime pulsado. Em lasers que possuem um espectro de ganho amn' longitudinais podem ser excitados s............

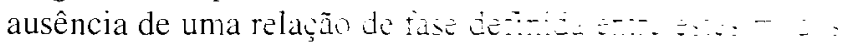
o laser opera continuamente. pois nà = interferência durante o batimento interno do med is. $\mathrm{P}$ - $-\ldots$ se uma diferença de fase fixa entre modos adjacentes é forçada, cria-se um padrão de interferência por batimento dos modo longitudinais, de forma que o laser deixa de emitir continuamente e passa a emitir pulsos muito curtos a uma taxa de repetição equivalente ao espaçamento modal. Quando o travamento de modos acontece, além do regime pulsado de funcionamento, o conteúdo de erro de fase dos modos diminui consideravelmente. Assim, o espectro do sinal do laser passa a conter um certo número de modos (pente de frequiências) limitado pela largura de banda de ganho. separados pela mesma distância. e com alta pureza espectral. Através da seleção apropriada de modos. pode-se fotodetetar o sinal do laser travado, obtendo-se, assim, sinais eletrònicos cuja as freqüências correspondem ao batimento das freqüência dos modos escolhidos.

Uma das maneiras de se criar uma referência de fase para os modos da cavidade do laser é através da modulação do laser em freqüências ou sub-frequências equivalentes ao espaçamento modal. Infelizmente, a separação entre modos de lasers semicondutores, por exemplo, é da ordem de centenas de $\mathrm{GHz}$. Além dos custos adicionais em se utilizar 
fontes que forneçam sinais nesta faixa de freqüência, o próprio laser não conseguiria responder a modulação. Uma maneira de contornar o problema é através da utilização de cavidades estendidas, que diminuem o espaçamento modal. A Fig. 8 mostra o diagrama esquemático de uma estrutura especial para travamento de modos. Trata-se de uma estrutura integrada OEIC composta de um região do tipo laser semicondutor DFB (distributed feedback), acoplada a um guia de onda passivo, cujo o comprimento é muito maior que o da região ativa DFB [8].

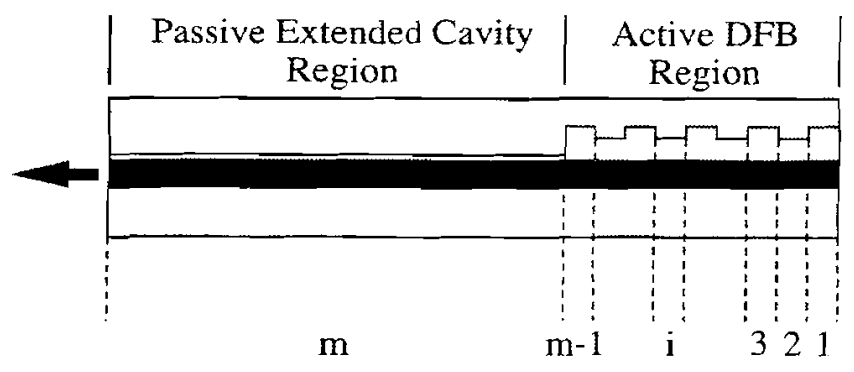

Figura 8. Região ativa DFB integrada com uma cavidade estendida passiva [8].

Normalmente, um laser DFB é fortemente seletivo, sendo considerado monomodo devido à sua estreita largura de banda de ganho. Contudo, ao se acoplar uma cavidade estendida à região $\mathrm{DFB}$, o espaçamento modal diminui consideravelmente, de forma que mais de um modo longitudinal passa a se encaixar dentro da banda de ganho da região ativa. Sob estas condições, o OEIC passa a emitir dois ou mais modos, cuja a distância espectral pode ser controlada através do comprimento da cavidade estendida. Através da modulação da corrente de alimentação do OEIC, pode-se obter o travamento de modos.

A Fig. 9 mostra o espectro eletrônico gerado após a fotodeteção do sinal de saída de uma estrutura OEIC, como a descrita acima, sob travamento de modos [8]. Neste caso. o comprimento da cavidade foi projetado para que a estrutura suportasse apenas dois modos longitudinais. A freqüência de modulação da corrente do OEIC correspondeu à metade da frequiência de espaçamento modal, ou seja. por volta de $7,38 \mathrm{GHz}$. Notar que a freqüência do sinal correspondente ao batimento dos dois modos sob travamento é de 14,76 GHz. Neste caso, a medida da densidade espectral de ruído de fase produz um valor melhor que $-90 \mathrm{dBc} / \mathrm{Hz}$ em $10 \mathrm{kHz}$ de offset.

Infelizmente, as técnicas mencionadas nos últimos parágrafos requerem equipamentos e componentes especiais que tornam os projetos ópticos e/ou eletrônicos complexos. caros e sensíveis. Uma outra alternativa simples para a geração de sinais, baseado em [9], utiliza o efeito de mistura de sinais em amplificadores ópticos semicondutores (SOA) [10]. Comparando-se os resultados conseguidos com a montagem descrita em [10] com os resultados de [9], pôdese demonstrar, experimentalmente. que SOAs em cascata são capazes de misturar com maior eficiência duas portadoras ópticas moduladas em frequências distintas e espaçadas de $8 \mathrm{~nm}$. Um analisador de espectro mostrou que a fotocorrente produzida após a filtragem de uma das portadoras ópticas continha componentes harmônicas correspondentes às frequências de soma e subtração das duas frequências modulantes das portadoras opticas individuais. Neste artigo, apresenta-se um método alternativo para a geração de sinais de RF e microondas em relação àquele que utiliza SOAs cascateados. Para minimizar o número de SOAs na montagem experimental, propōe-se uma configuração onde se utiliza SOAs com realimentação óptica (FB-SOA). Nesta configuração com realimentação, pode-se assegurar um estado mais profundo de saturação do dispositivo, aumentando-se os níveis de potência dos componentes de freqüência que podem ser gerados.

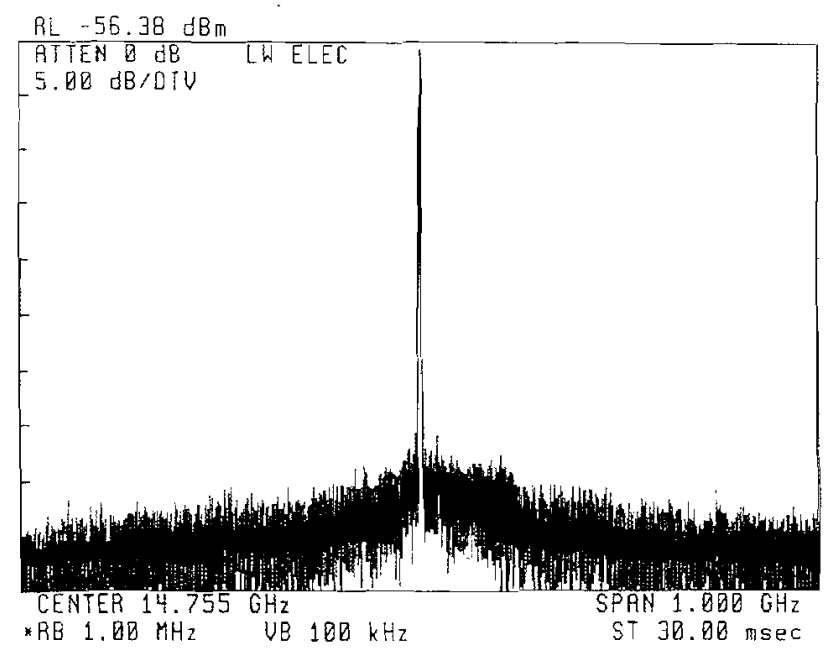

Figura 9. Espectro de sinal eletrônico obtido após fotodeteção do sinal de saída de um OEIC sob travamento de modos [8].

A fim de se compararem os diferentes esquemas de geração/conversão de sinais utilizando SOAs, realizou-se uma série de experimentos onde os SOAs foram utilizados em configurações cascateadas e realimentadas. Primeiramente, um experimento foi realizado para demonstrar as propriedades de mistura de um SOA (1SOA), quando o mesmo é submetido à aplicação de dois sinais ópticos de comprimentos de onda distintos, modulados em diferentes frequiências [10]. Sob as mesmas condições, um segundo SOA é adicionado ao aparato experimental, em série com o primeiro, de forma a se observar o efeito da utilização cascateadas de SOAs (2SOA) na mistura de sinais [10]. A seguir, um experimento com o SOA realimentado opticamente foi implementado (FB-SOA), utilizando-se os mesmos sinais ópticos modulados. Neste caso particular, foi possível observar que o método FB-SOA pode oferecer um desempenho similar àquele do esquema 2-SOA. Contudo uma investigação mais detalhada do aparato experimental do FB-SOA revelou que este método pode superar os resultados obtidos para o 2SOA. Uma maneira encontrada de se comprovar esta hipótese foi por meio do acréscimo de um segundo SOA em cascata (série) com o FB-SOA. Neste caso, reproduz-se o experimento 2-SOA, porém, utilizando-se o primeiro SOA realimentado. Como esperado, observou-se um desempenho superior na mistura de sinais em relação aos resultados obtidos com o experimento de simples cascateamento dos amplificadores ópticos (2-SOA). 


\section{TEORIA E EXPERIMIENTOS}

A Fig. 10 ilustra o mecanismo de mistura para duas portadoras ópticas moduladas em amplitude que estão sendo simultaneamente acopladas a um SOA. Uma das portadoras é gerada por um laser transmissor (TO), que opera no comprimento de onda $\lambda_{\mathrm{TO}}\left(\mathrm{f}_{1}=\mathrm{c} / \lambda_{\mathrm{TO}}\right.$, onde $\mathrm{c}$ é a velocidade da luz) e é modulada numa freqüência $f_{\mathrm{TO}}$. A outra é produzida por um laser oscilador local (LO), que opera no comprimento de onda $\lambda_{\mathrm{LO}}\left(\mathrm{f}_{2}=\mathrm{c} / \lambda_{\mathrm{LO}}\right)$ e é modulada numa frequiência $f_{\mathrm{LO}}$. Se a potência óptica acoplada ao amplificador óptico semicondutor for suficiente para saturálo profundamente, a população de portadores na região de ฐanho do SOA diminui significativamente, resultando em uma considerável redução do ganho do amplificador. Contudo, a condição de saturação profunda torna o ganho do SOA fortemente sensível a qualquer variação do número de fótons no interior de sua cavidade. Desta forma, o SOA responde de forma sincronizada em relação às flutuações da potência óptica acoplada, originada pela modulação de intensidade óptica das portadoras. Portanto, como conseqüência das flutuações, o ganho óptico é simultaneamente modulado em ambas as freqüências de modulação das portadoras ópticas $\mathrm{f}_{\mathrm{TO}}$ e $\mathrm{f}_{\mathrm{LO}}$, de tal forma que o SOA torna-se incapaz de distinguir e separar cada uma das portadoras ópticas de suas próprias componentes de frequiência. Como resultado, a modulação do ganho do SOA induz um amplificação modulada das portadoras ópticas nas freqüências $f_{T O}$ e $f_{L O}$. Este efeito, conhecido como saturação cru:ada de ganho, é o principal responsável pelas características de mistura do SOA.

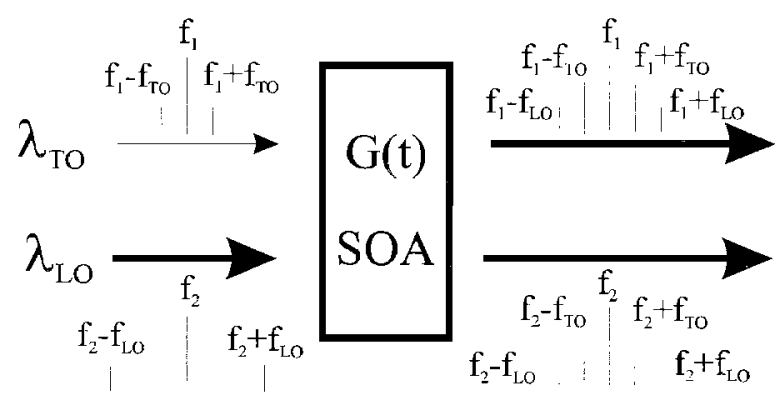

Figura 10. Diagrama de bloco mostrando a propriedade de mistura do SOA.

Agora, se o sinal óptico do LO é filtrado na saída após o SOA e o sinal resultante for fotodetetado, a fotocorrente irá conter harmônicas nas freqüências $f_{T O}+f_{L O}$ e $f_{T O}-f_{L O}$. Escolhendo-se corretamente $\mathrm{f}_{\mathrm{TO}}$ and $\mathrm{f}_{\mathrm{LO}}$, ambos os sinais, convertidos para cima $\left(f_{T O}+f_{L O}\right)$ e para baixo $\left(f_{T O}-f_{L O}\right)$, podem estar localizados dentro de um intervalo de frequiência específico, que pode variar entre RF, microondas e, potencialmente, até ondas milimétricas.

Em [10], demonstrou-se que o uso combinado de SOAs em cascata com o propósito de geração de portadoras de RF e/ou microondas assegura a saturação do ganho. Portanto, o efeito da saturação do ganho é alcançado de maneira mais rápida e eficiente do que pela modulação da corrente do SOA, mesmo para sistemas onde a potência óptica de LO e TO são baixas. A Fig. 11 mostra o diagrama de bloco da configuração experimental de SOAs em cascata (2-SOA). O laser TO é diretamente modulado com a frequência de modulação $\mathrm{f}_{\mathrm{TO}}$ e o sinal do laser LO é modulado externamente, via um modulador óptico, com a freqüência de modulação $f_{L O}$. Ambos os sinais modulados passam então através de um acoplador óptico a fibra, balanceado, e são injetados no interior da região ativa do SOAl. Na sequiência, o sinal gerado na saída do SOAl é acoplado ao SOA2. Como o sinal na entrada do SOA2 já foi amplificado, garante-se um maior nível de potência acoplado a este amplificador, maximizando-se a sua saturação, e, com isto, justificando-se uma possível melhoria no processo de mistura entre os dois sinais ópticos. A seguir, o sinal resultante da dupla amplificação localizado na saída do SOA2 passa através de um filtro óptico centrado em $\lambda_{\text {TO }}$ e é fotodetetado. A fotocorrente produzida é amplificada e acoplada a um analisador de espectro. Controles de polarização são usados para casar a polarização dos feixes dos lasers com a polarização das frentes de onda do SOA. Isoladores evitam reflexões indesejadas e acoplamentos entre SOAs.

A Fig. 12 mostra o diagrama de bloco do experimento com SOA realimentado ópticamente (FB-SOA). Em comparação com a Fig. 11. as modificações na configuração experimental localizam-se após o acoplador a fibra A, agora representado como acoplador à fibra A1. Ambos os sinais modulados passam através de A1 e A2 e são acoplados ao SOA. O sinal na saída do SOA é realimentado opticamente via A3 para nova amplificação e melhoria do processo de mistura. Seguindo agora no sentido inverso, o sinal óptico passa mais uma vez por A2 e é acoplado à um filtro óptico centrado em $\lambda_{\text {TO }}$. A fotocorrente produzida após detecção é amplificada e acoplada a um analisador de espectro. Da mesma forma que no caso do experimento SOA-2, controles de polarização são utilizados para o casamento de polarização das frentes de onda dos feixes dos lasers e dos feixes no interior do SOA. O uso de isoladores previne acoplamento de emissões e reflexões indesejadas nos lasers e SOA.

A Fig. 13 mostra uma variação do diagrama de bloco experimental da Fig. 12. Em comparação com a Fig. 12. a Fig. 13 apresenta um segundo SOA colocado em série com - SOA realimentado (após a saída de A2). O objetivo da colocação deste SOA adicional é compensar as perdas de sinal nos acopladores utilizados na Fig. 12, mais especificamente. nos acopladores A2 e A3, e permitir uma comparação mais precisa entre as técnicas 2-SOA e FBSOA. Assim, mais uma vez, os sinais modulados do lasers passam através dos acopladores à fibra A1 e A2 e são acoplados ao SOA. O sinal na saída do SOAl é realimentado, passa mais uma vez por A2 e é acoplado à cavidade do SOA2. Após este estágio, a luz passa por um filtro óptico centrado em $\lambda_{\text {TO }}$. Finalmente, o sinal óptico é fotodetetado e a fotocorrente observada em um analisador de espectro. 


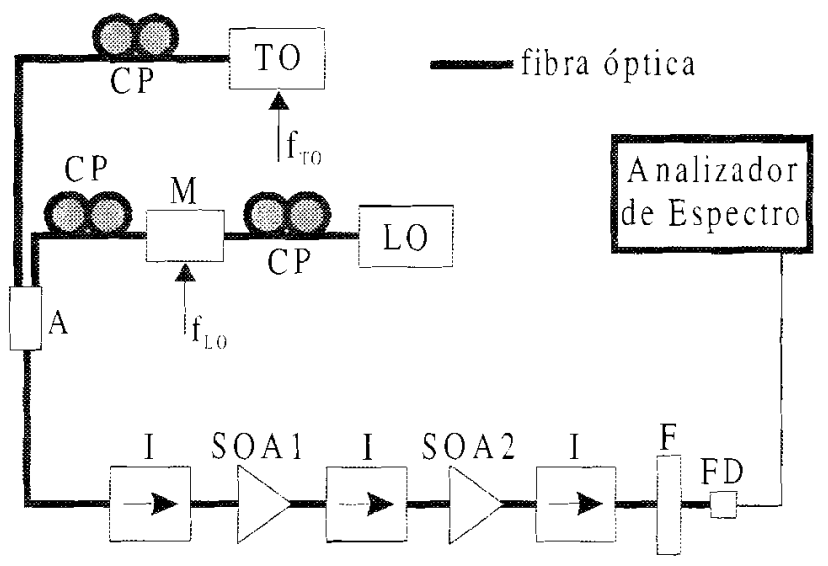

Figura 11. Diagrama de bloco da configuração de SOAs em cascata. TO: laser transmissor; LO: laser local; M: modulador óptico; CP: controle de polarização; A: acoplador à fibra; FD: fotodetetor; SOA: amplificadores ópticos semicondutores; I: isoladores; F: filtro óptico:

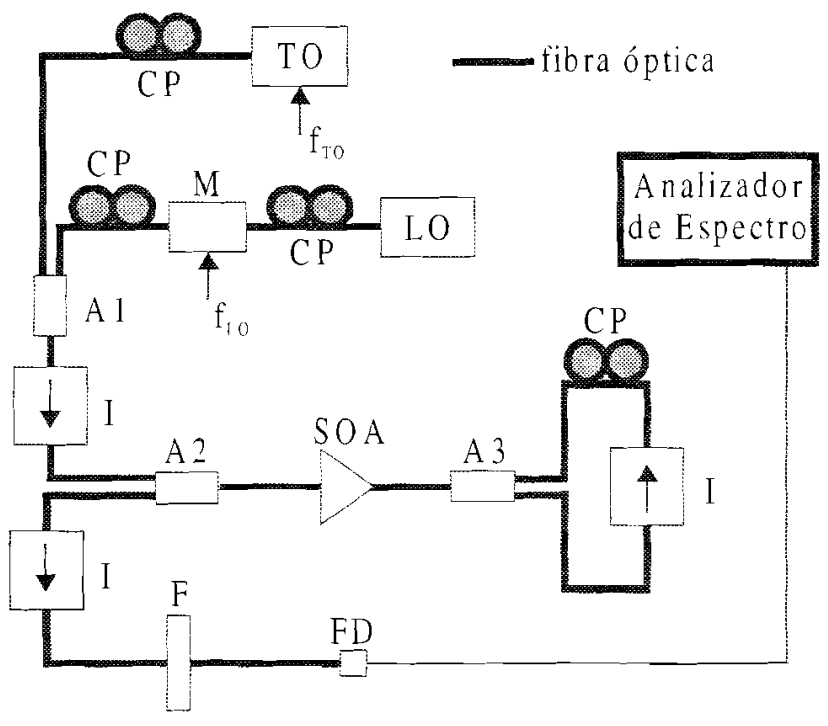

Figura 12. Diagrama de bloco da configuração FB-SOA1.

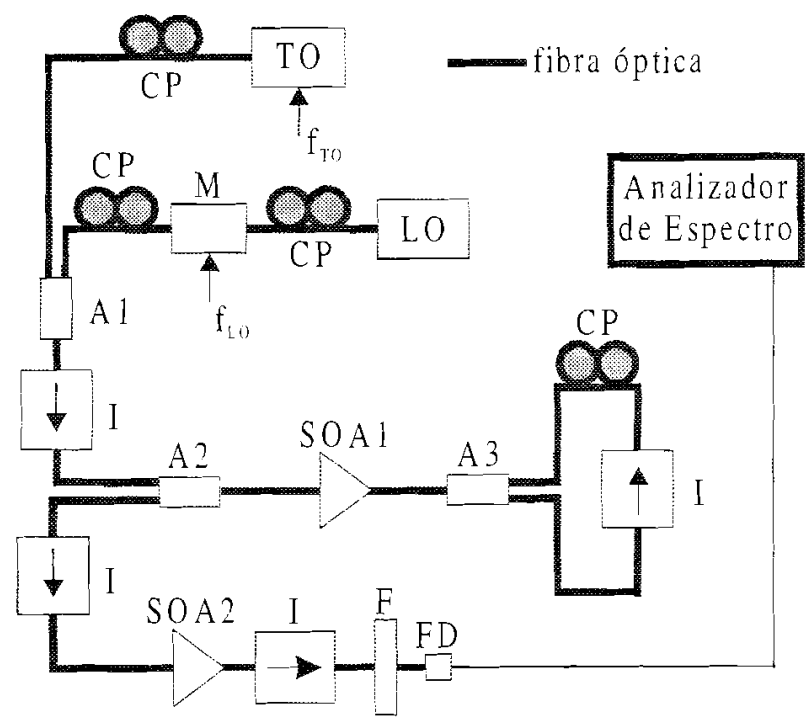

Figura 13. Diagrama de bloco da configuração FB-SOA-2.

\section{RESULTADOS EXPERIMENTAIS}

A fonte laser $\mathrm{TO}$ foi um laser semicondutor em cavidade externa (E-Tek Ind.) operando em um único comprimento de onda $\lambda_{\mathrm{TO}}=1548,95 \mathrm{~nm}$. Sua corrente de polarização foi de $120 \mathrm{~mA}$. Este laser foi modulado diretamente em $\mathrm{f}_{\mathrm{TO}}=250 \mathrm{MHz}$ por um gerador de sinais Wavetek 2500A. A fonte laser LO foi um laser semicondutor em cavidade externa sintonizável (Photonetics) operando em um comprimento de onda distante $8.1 \mathrm{~nm}$ de $\lambda_{\text {TO }}$, ou seja, em $\lambda_{\text {LO }}=1540,85 \mathrm{~nm}$. A corrente de polarização de LO foi de $60 \mathrm{~mA}$ e seu sinal de saída foi modulado por um modulador externo (Sumitomo Inc) em $\mathrm{f}_{\mathrm{LO}}=400 \mathrm{MHz}$ via um gerador de sinais HP 8657A. Durante os experimentos, os SOAs (E-Tek Ind.) foram polarizados com $120 \mathrm{~mA}$. Um fotodetetor de $300 \mathrm{kHz}-6 \mathrm{GHz}$ (HP 83411A) foi usado para detectar os sinais ópticos após um filtro óptico de largura de banda de $6 \mathrm{~nm}$ centrado em $1550 \mathrm{~nm}$. A Fig. 14(a) mostra o espectro óptico para ambos os sinais antes do filtro óptico. Os marcadores do analisador de espectro óptico indicam a distância de $8,1 \mathrm{~nm}$ entre as portadoras ópticas.

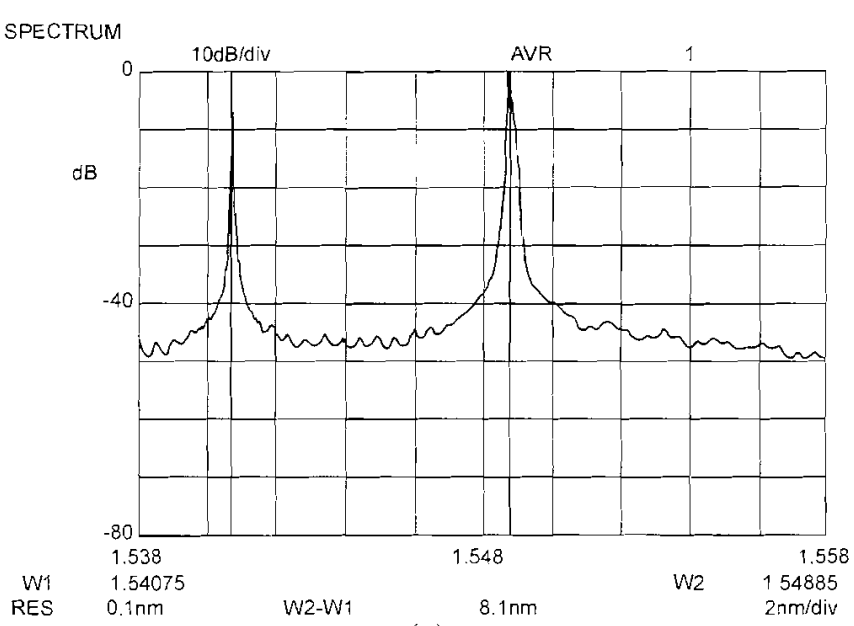

SPECTRUM

(a)

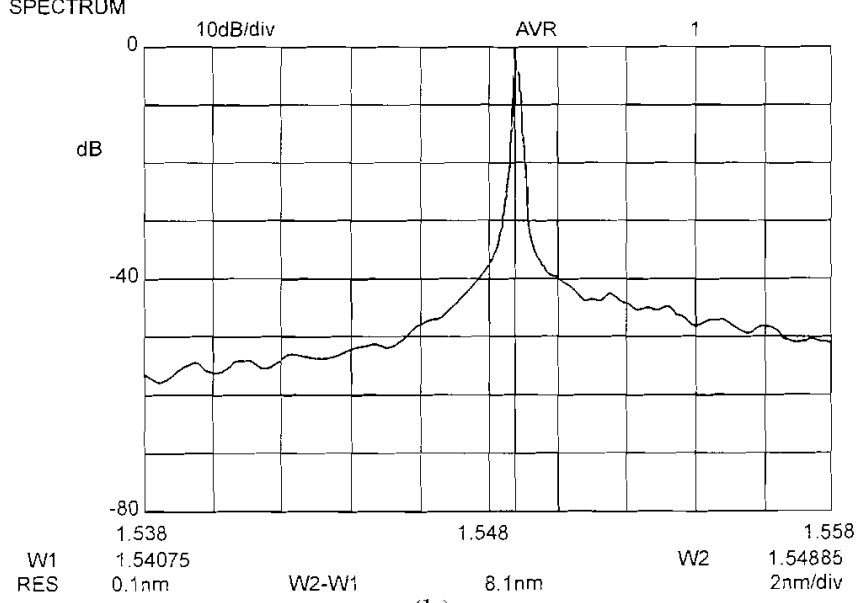

(b)

Figura 14. Espectro óptico para os sinais TO $(1540.75 \mathrm{~nm})$ e LO (1548.85 nm) antes (a) e depois (b) da filtragem óptica.

A filtragem óptica, Fig. 14(b), foi usada na entrada do fotodetetor como uma forma a prevenir alguma contribuição excessiva de ruído do sinal de LO (e. g., ruído de 
intensidade - relative intensity noise. RIN) e dos SOAs (isto é, emissão espontânea amplificada) na geração dos sinais convertidos para cima e para baixo. Limitações na resposta de modulação direta do laser TO impossibilitaram freqüências de modulação das portadoras ópticas maiores do que estas listadas acima.

Referindo-se à Fig. 11, a fim de observar as implicações do emprego de SOAs em cascata na geração de sinais de RF e microondas e ilustrar o mecanismo de mistura dos SOAs, primeiramente, realizou-se $O$ experimento com apenas um SOA (SOA 1 ou 2 na Fig. 11). A Fig. 15 mostra a medida da potência espectral do sinal eletrônico obtido após o fotodetetor, onde é possível observarem-se seis componentes de frequiência principais. Os níveis de sinal das harmônicas que correspondem às freqüências de modulação de TO e LO são de, aproximadamente, $-46 \mathrm{dBm}$ e $-62 \mathrm{dBm}$, respectivamente. Uma segunda harmônica para $f_{\text {To }}$ aparece na Fig. 6, com cerca de $-58 \mathrm{dBm}$, fruto da modulação direta do laser e das características da fonte de modulação. Estes dois fatores também são responsáveis pelo aparecimento do sinal observado próximo a $560 \mathrm{MHz}$, resultado do batimento de harmônicas superiores das frequiências de modulação. Finalmente. as componentes resultantes da mistura em 650 $\mathrm{MHz}$ e $150 \mathrm{MHz}$, apresentaram níveis de potência sensivelmente menores que os valores medidos para as freqüências de modulação (cerca de $35 \mathrm{~dB}$ menor que a harmônica de modulação de TO). Este resultado era esperado, já que a potência óptica acoplada ao SOA foi insuficiente para sua saturação profunda, o que implicou em um pobre efeito de mistura.

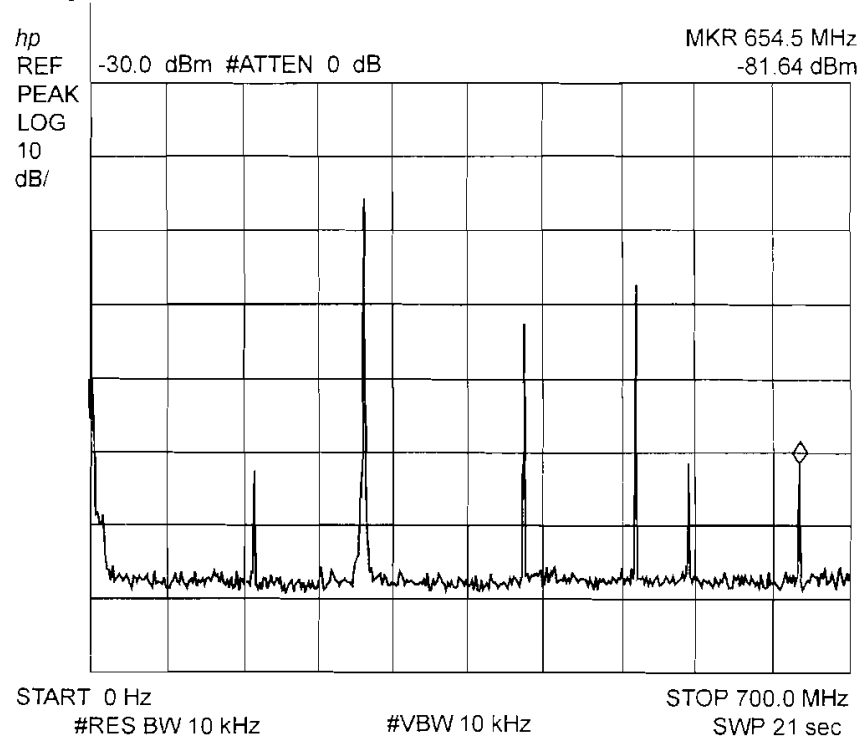

Figura 15. Espectro do sinal de saída do fotodetetor para o sistema 1-SOA.

A Fig. 16 mostra o espectro de potência após a adição do SOA2. O uso de SOAs em cascata tende a melhorar o efeito de mistura. uma vez que uma condição de saturação profunda pode ser alcançada mais efetivamente. Como resultado, o segundo SOA amplifica mais dinamicamente as harmônicas menos intensas do sinal óptico. Comparando-se a Fig. 16 com a Fig. 15, pode-se observar que as componentes convertidas para cima e para baixo foram amplificadas em torno de 10 a $12 \mathrm{~dB}$ após o SOA2 (para cerca de $-69 \mathrm{dBm}$ e $-73 \mathrm{dBm}$. respectivamente). Então, a adição de novos SOAs poderá aumentar o nível de potência dos sinais convertidos para cima e para baixo, principalmente para cima. que são de maior interesse para aplicações em sistemas que utilizam RF e/ou microondas.

Referindo-se à Fig. 12, a Fig. 17 mostra o espectro de potência da fotocorrente para o experimento FB-SOA. Apesar do uso de apenas um SOA no arranjo de realimentação óptica, as componentes convertidas para cima e para baixo estão presentes com níveis de potência maiores (-69 $\mathrm{dBm}$ and $-72 \mathrm{dBm}$, respectivamente) que os níveis obtidos no experimento com 1-SOA.

Comparando as Fig. 16 e 17, é possível verificar que o nível de sinal da harmônica convertida para cima é praticamente o mesmo nas duas figuras. Resultado semelhante também foi observado comparando-se as freqüências fundamentais em 250 e $400 \mathrm{MHz}$. Assim, podese concluir que a configuração FB-SOA tem o potencial de apresentar desempenho superior quando comparado ao caso 2-SOA, uma vez que uma quantia considerável de potência óptica foi perdida nos acopladores à fibra. De fato, a montagem para o experimento FB-SOA teve perdas em excesso de $6 \mathrm{~dB}$ em $\mathrm{A} 2$, e de $6 \mathrm{~dB}$ em A3, em comparação com o experimento 2-SOA. Modificações na configuração experimental poderiam implicar num gerenciamento melhor da potência do sistema. Por exemplo, um circulador óptico poderia substituir o acoplador à fibra $\mathrm{A} 2$, fornecendo $6 \mathrm{~dB}$ extras de potência óptica para os processos de saturação do SOA e. também, fotodeteção. Uma outra modificação seria a substituição do acoplador A3 por um espelho, resultando num acréscimo suplementar de $6 \mathrm{~dB}$ para o efeito de saturação cruzada do SOA.

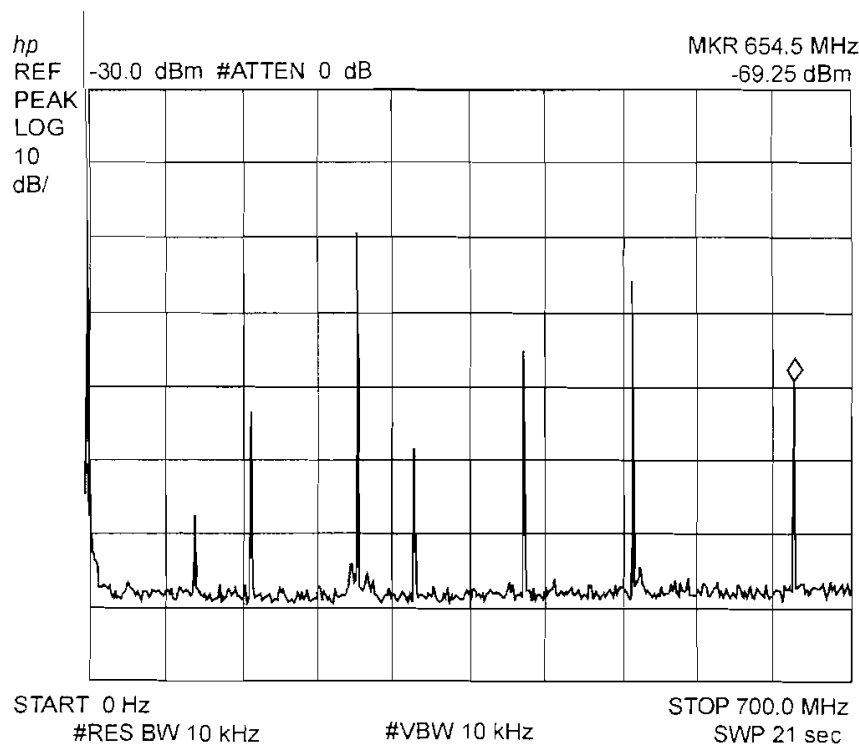

Figura 16. Espectro do sinal de saída do fotodetetor para o sistema 2-SOA.

Referindo-se à Fig. 13, a Fig. 18 mostra o espectro de potência da fotocorrente para o experimento FB-SOA2, onde são utilizados dois SOAs: um SOA com realimentação óptica e outro em série com a saída do primeiro. Esta configuração procura dar uma idéia do aumento em nivel de sinal que as frequiências convertidas para cima e para baixo 
poderiam ter caso as perdas introduzidas por A2 e A3 pudessem ser compensadas. Como pode ser observado na Fig. 18, as componentes convertidas para cima e para baixo possuem níveis de potência maiores $(-63 \mathrm{dBm}$ and $-70 \mathrm{dBm}$, respectivamente) do que aqueles medidos no experimento FB-SOA. Isto comprova uma melhoria ainda maior no desempenho. Se, agora, as Fig. 16 e 18 são comparadas, observa-se que o nível de sinal da harmônica convertida para cima é cerca de $6 \mathrm{~dB}$ maior que o resultado obtido no experimento 2-SOA. Este resultado sugere que, de fato, se as perdas nos acopladores pudessem ser eliminadas ou minimizadas, o FB-SOA poderia, realmente, oferecer desempenho superior.

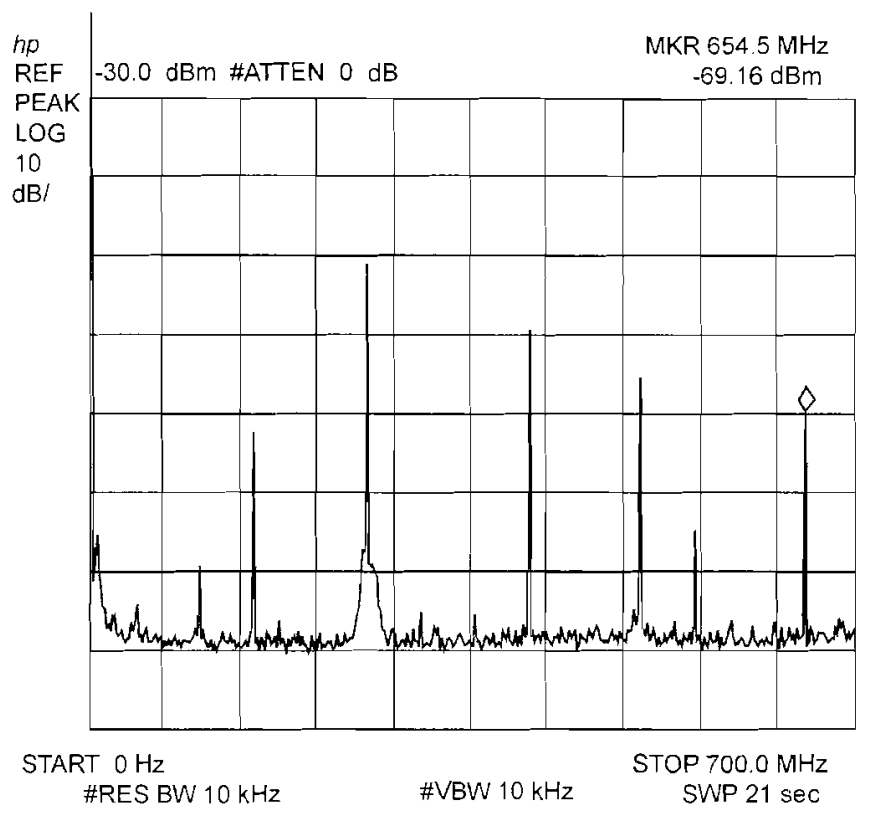

Figura 17. Espectro do sinal de saída do fotodetetor para o experimento FB-SOA.

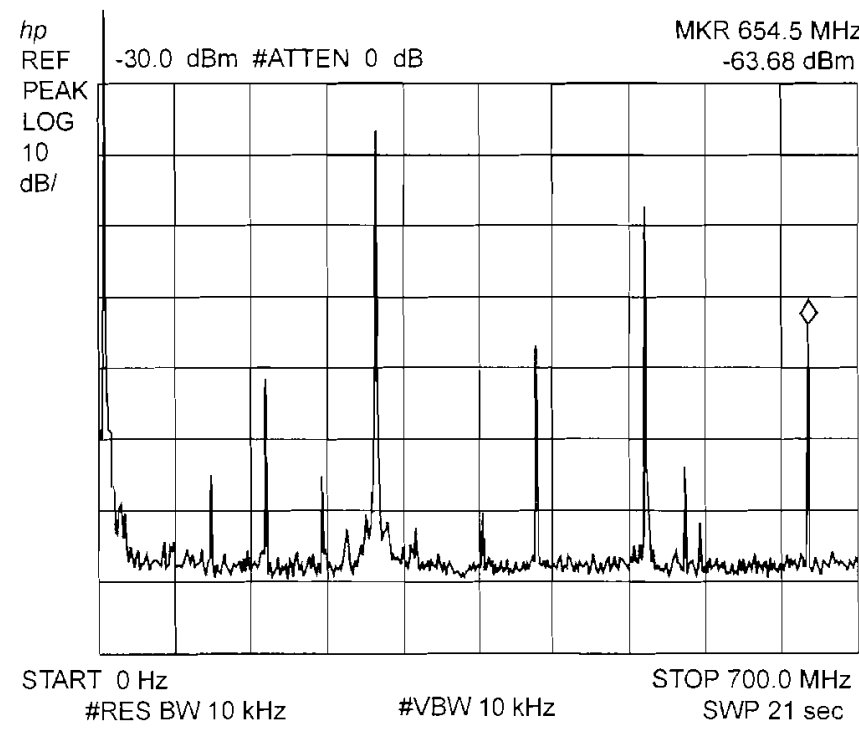

Figura 18. Espectro do sinal de saída do fotodetector para o experimento FB-SOA2.

A medida do ruído de fase do sinal convertido para cima é melhor que $-70 \mathrm{dBc} / \mathrm{Hz}$ para $100 \mathrm{kHz}$ de offset. Esta medida foi limitada pelo de ruído de fundo do analisador de espectro. A amplitude das harmônicas mostrou-se sensível às oscilações de amplitude do laser (ruído RIN) e à interferência de fase no caminho de realimentação devido às variações térmicas. Também, observou-se oscilações de freqüência. Portanto, a qualidade do sinal convertido depende não apenas da melhoria da configuração experimental, mas também da qualidade das fontes ópticas empregadas.

\section{CONCLUSÕES}

Neste trabalho, demonstrou-se a possibilidade de melhorar as propriedades de mistura de SOAs, no intuito de gerar sinais nas faixas de frequiência de RF e microondas, utilizando-os em configurações com realimentação óptica. Neste caso, o uso de apenas um SOA realimentado opticamente tem o potencial de melhorar os níveis de sinal para as componentes de freqüencia convertidos para cima e para baixo, em comparação com os resultados obtidos em experimentos com dois SOAs cascateados. A melhoria de desempenho pode ser alcançada se a configuração experimental FB-SOA original for remodelada para minimizar perdas nos acopladores. Numa tentativa de compensação das perdas, observou-se que cerca de $6 \mathrm{~dB}$ de aumento no nível de potência do sinal convertido para cima pode ser alcançado se um segundo SOA for colocado em série com o SOA opticamente realimentado.

Agradecimentos ao CEPID-FAPESP, CNPq, CAPES, FAEP/UNICAMP e MCT-Pronex pelo suporte financeiro.

\section{REFERÊNCIAS}

[1] S. L Woodward. and S. Ariyavistakul, "Transporting CDMA signals over an analog optical link", IEEE Trans. Vehic. Teclinol., vol. 48, no. 4. pp. 1033-1038, 1999.

2] R. Ohmoto, H. Ohtsuka, and H. Ichikawa, "Fiber-optic microcell radio systems with spectrum delivery scheme", IEEE Trans. Vehic. Technol., vol, 11, no. 7, pp. 1108-1117. 1993.

[3] J. E. Bowers, A. C. Chipalosky, and S. Boodaghians, "Longdistance fiber-optic transmission of c-band microwave signals to and from a satellite antenna", IEEE J. Lightwave Technol., vol. 5, no. 12. pp. 1733-]741, 1987.

[4] R. Olshansky. V. Lanzisera, and P. Hill, "Subcarrier multiplexed lightwave systems for broad-band distribution". IEEE J. Lightwave Technol., vol. 7. no. 9, pp. 1329-1342, 1989.

[5] R. T. Ramos, and A. J. Seeds, "Fast heterodyne optical phase-lock loop using double quantum well laser diodes", Electron. Lett., vol. 28, no. 1. pp. 82-83, 1992.

[6] C. Walton, A. C. Bordonalli, and A. J. Seeds. "High performance heterodyne optical injection phase-lock loop using wide linewidth semiconductor lasers", IEEE Photon. Technol. Lett., vol. 10, no. 3, pp. 427-429, 1998

[7] L. Goldberg, H. F. Taylor, and J. F. Weller, "FM sideband injection locking of diode lasers", Electron. Lett., vol. 18, no. 23. pp. 1019-1020, 1982.

[8] A. C. Bordonalli, B. Cai, A. J. Seeds, and P. J. Williams, "Generation of microwave signals by active mode locking in a gain bandwidth restricted laser structure", IEEE Photon. Technol. Lett., vol. 8, no. 1. pp. 151-153, 1996

[9] W. Shieh, S. X. Yao. G. Lutes, and L. Maleki, "An alloptical microwave mixer with gain", OFC'97 Technical Digest. ThGl. pp 263-264. 1997. 
[10] A. C. Bordonalli, A. D. Neto. C. R. Lima, and E. Conforti. "Microwave signal generation by mixing of modulated optical carriers in saturated semiconductor optical amplifiers", IEEE/MTT-S/SBMO International Microwave and Optoelectronics Conference, Rio de Janeiro. Brazil, pp. $417-420,1999$.

\begin{abstract}
Aldário C. Bordonalli nascido em Ribeirão Preto. SP. em 1967. Recebeu o diploma de Bacharel em Física Aplicada pelo Instituto de Física da Universidade Estadual de Campinas, en 1989. Obteve o grau de Mestre na área de telecomunicações (comunicações ópticas), pela Faculdade de Engenharia Elétrica e de Computação da Universidade Estadual de Campinas, em 1992. Em 1996. concluiu o doutoramento pelo Department of Electronic and Electrical Engineering da University College London. Londres. Inglaterra. na mesma área de atuação. Atualmente, é professor Assistente Doutor do Departamento de Microondas e Óptica da Faculdade de Engenharia Elétrica e de Computação da Universidade Estadual de Campinas. Suas principais áreas de pesquisas são: lasers semicondutores em cavidades externas e estendidas: técnicas para sincronização de frequêencia óptica de lasers semicondutores; amplificadores ópticos: geração/conversão optoeletrônica de sinais; sistemas e redes WDM e DWDM: sistemas de remodulação, apagamento e reformatação de sinais em sistemas ópticos; e sistemas de microonda e RF. Possui mais de 20 trabalhos publicados em conferências e revistas nacionais e internacionais.
\end{abstract}

Jéferson Luiz Benitez nasceu en Foz do Iguaçu, PR, em 1975. Graduou-se em Engenharia Elétrica em 1997 pela Universidade Estadual de Mato Grosso. Atualmente, desenvolve pesquisa na área de comunicaçōes ópticas como aluno do programa de Mestrado da Faculdade de Engenharia Elétrica e de Computação da Universidade Estadual de Campinas. Suas principais áreas de interesse são a geração/conversão optoeletrônica de sinais e os amplificadores ópticos semicondutores.
Joselan Álvaro Guimarães nasceu São Paulo. SP. em 1973. Formou-se em Engenharia Elétrica pela Universidade Federal de Pernambuco em 1998. Atualmente. é aluno do programa de Mestrado da Faculdade de Engenharia Elétrica e de Computação da Universidade Estadual de Campinas. Dentro da área de comunicações ópticas, seus interesses concentram-se na geração/conversão optoeletrônica de sinais. em técnicas para sincronização de freqüência óptica de lasers semicondutores, em amplificadores ópticos e em sistemas de remodulação, apagamento e reformatação de sinais ópticos.

Christiano Mello Gallep nasceu en Sorocaba, SP. em 1974. É Engenheiro Elétrico, formado pela Faculdade de Engenharia Elétrica e de Computação da Universidade Estadual de Campinas em 1997. onde obteve também o grau de Mestre em 1999. Atualmente é doutorando na mesma instituição, trabalhando com simłlações e aplicações de amplificadores ópticos semicondutores. Além de assuntos relacionados a comunicações ópticas e seus dispositivos, tem interesses voltados para o ensino de engenharia e para o desenvolvimento e aplicações da Teoria de Biofótons.

Evandro Conforti nasceu em S. J. Rio Preto, SP, em 1947. Graduou-se em Engenharia Eletrônica pelo Instituto Tecnológico da Aeronáutica. 1970. Obteve o grau de Mestre pela University of Toronto. Canada. em 1978. Em 1983. concluiu o doutoramento pela Faculdade de Engenharia Elétrica e de Computação da Universidade Estadual de Campinas. Desde 1981. o Prof. Conforti é membro do corpo docente da Universidade Estadual de Campinas. De 1984 a 1987, ele foi diretor da Faculdade de Engenharia Elétrica e de Computação, onde atualmente ocupa o cargo de professor titular junto ao Departamento de Microondas e Óptica. Entre os anos de 1992 e 1994, foi professor visitante da University of Illinois em Urbana-Champaign. Recebeu, em 1988.0 "Prêmio Governador do Estado - Invento Brasileiro" e, em 198: o "Prêmio de Pesquisa da Unicamp". Possui seis patentes e é coautor de um livro. além de ter publicado mais de 80 trabalhos em conferências e revistas nacionais e internacionais. Orientou, até hoje, três teses de doutorado e mais de dezessete dissertações de mestrado. Suas principais areas de interesse e pesquisa incluem os amplificadores ópticos semicondutores e o chaveamento totalmente óptico. 